\title{
Philosophiques
}

\section{Le phénomène cognitif comme éco-système chez Piaget}

\section{Robert Plante}

Volume 4, numéro 2, octobre 1977

Philosophie et psychologie

URI : https://id.erudit.org/iderudit/203074ar

DOI : https://doi.org/10.7202/203074ar

Aller au sommaire du numéro

Éditeur(s)

Société de philosophie du Québec

ISSN

0316-2923 (imprimé)

1492-1391 (numérique)

Découvrir la revue

Citer cet article

Plante, R. (1977). Le phénomène cognitif comme éco-système chez Piaget.

Philosophiques, 4(2), 245-249. https://doi.org/10.7202/203074ar d'utilisation que vous pouvez consulter en ligne.

https://apropos.erudit.org/fr/usagers/politique-dutilisation/ 


\title{
LE PHENOMÈNE COGNITIF COMME ECO-SYSTÈME CHEZ PIAGET
}

\author{
par Robert Plante
}

Piaget est sans doute l'un des penseurs contemporains les plus "décevants" qui soient. Je vois deux clés qui puissent le rendre plus facilement pénétrable ; la première nous est fournie par Piaget lui-même, et il s'agirait alors de passer par la philosophie, ou d'en venir, pour accéder au niveau de son projet et à son sens. Dans ce court exposé, je vais risquer de vous proposer la deuxième, sous la forme d'une définition de l'épistémologie génétique, qui rend, à mon avis, plus claires les affirmations centrales qui ont valu à Piaget tant de résistances aussi bien du côté des philosophes que de celui des biologistes ou des mathématiciens.

Il s'agit de bien indiquer que son point de départ n'est réductible à aucune approche traditionnelle du problème de la connaissance, même si, pour le bien situer, il faille faire appel à toutes. L'épistémologie génétique m'apparaît ainsi comme une métbode d'interprétation des relations dynamiques à l'intérieur de l'écosystème cognitif. Piaget introduit de cette façon en épistémologie, et de façon abrupte, ce que la biologie a mis cent ans à préciser. Lorsque Haeckel, en 1866, a proposé le terme "écologie " pour désigner la science du " milieu ", il semblait bien n'ajouter qu'une branche à une science déjà dans son essor de spécialisation. Ce n'est que tardivement, et au moment où la conversion culturelle le permit, qu'on s'est rendu compte que le couple organisme-milieu se comportait comme un système ou un organisme en soi, redevable d'un traitement comme tel. L'écologiste, en ce sens, est moins intéressé aux définitions du vivant et du milieu qu'à la réalité complexe des interactions qui maintiennent le système global dans son état d'équilibre ou de déséquilibre.

Le constructivisme piagétien ne fait que transposer (ou généraliser) sur le plan cognitif cette interprétation de la nature. À la 
dichotomie traditionnelle sujet-objet, où chaque terme doit recevoir une explication qui rende compte de la relation cognitive, il substitue l'unité d'un continuum dont l'extension doit couvrir le monde total des objets dans un sens (extension spatiale) et la totalité du sujet dans l'autre (extension temporelle). D'où la nécessité, d'une part, d'enraciner la logique dans les manipulations sensorimotrices, et, d'autre part, de trouver les invariants structuraux et fonctionnels qui circulent de l'organique au logique.

Je ne veux citer que deux textes pour illustrer cette totalisation. Premièrement :

"L'expérience logico-mathématique (. . ) consiste à agir sur les objets mais avec abstraction des connaissances à partir de l'action et non plus des objets eux-mêmes. (.. .) l'expérience porte sur la liaison entre les caractères introduits par l'action dans l'objet ${ }^{\prime}$.

Deuxièmement, à l'intelligence-faculté il substitue l'intelligenceprocessus (il vaudrait mieux toujours dire: intelliger est un processus, comme Bertalanffy qui préférait substituer à "grenouille ", "ce qui est grenouillant"):

"Sous ce mot (intelligence) un peu vague et assez dangereux, il faut entendre précisément le fonctionnement des systèmes opératoires issus de l'action" ".

Car c'est toujours à l'intérieur des actions (interactions) que sont joints en un tout l'organisme et le milieu. Les mots clés sont donc solidairement: opératoire, interactionniste, génétique, qui, sans jamais rien exclure des objets, permettent de relier le système cognitif à tout système (thèse du volume Biologie et connaissance) et de le traiter comme un éco-système.

Une telle façon de voir oblige à plus de seconversion qu'on ne le pense généralement et, surtout, permet une lecture plus correcte des thèses piagétiennes fondamentales. Je me bornerai également ici à ne présenter brièvement que deux exemples. Voyons d'abord le cas de ce que Piaget appelle "le mythe de l'origine sensorielle des connaissances ${ }^{3}$. Ce qu'il entend par théorie de l'origine sensorielle, qu'il fait remonter à Aristote, dans un sens qui n'est d'ailleurs pas aristotélicien, c'est le fait d'affirmer que les contenus de

1. Piaget, Jean, Psycbologie et épistémologie, Gallimard, Paris, 1970, p. 89.

2. Ibid., p. 100.

3. PlaGeT, J., Psychologie et épistémologie, pp. 80-109. 
connaissances sont livrés " en tant que percepts " d'où nous tirons les concepts. Si telle est la vérité, nous aurons isolé là un élément du problème en essayant de le traiter en soi comme antérieur au système sujet-objet, et le savoir en serait, à toute fin pratique, ramené à une donnée dans les choses, et toute sa genèse ne serait que reconstruction. Nous reconnaissons bien là le reproche constant qu'Aristote fait à Platon, ce qui explique que les critiques de Piaget contre les "réalismes" frappent surtout le platonisme. À cette vue, Piaget oppose l'approche systémique :

"Nos connaissances ne proviennent ni de la sensation ni de la perception seules (nous pouvons lire : du milieu seul), mais de l'action entière dont la perception ne constitue que la fonction de signalisation. Le propre de l'intelligence n'est pas, en effet, de contempler mais de "transformer ", et son mécanisme est essentiellement opératoire $n^{4}$.

Le passage, probablement inconscient ici, de la sensation à l'intelligence indique bien la perspective : au niveau d'un éco-système, la relation sujet-objet ne permet pas d'isoler des termes ou d'aligner des étapes, mais seulement d'emboîter des cercles qui ont des propriétés identiques : et ce qui s'emboîte, ce sont des types d'actions et de transformations : " on ne connait un objet qu'en agissant sur lui et en le transformant".

Voici le deuxième exemple: si la notion d'éco-système permet bien de comprendre l'opposition piagétienne à la philosophie grecque ou de type réaliste parce qu'elle postulerait une structuration antécognitive, ou un savoir donné dans et avec le réel, cette même notion justifie davantage le rejet presque acharné de toute "l'illusion" philosophique postcartésienne. Après le Cogito, encore une fois, et plus radicalement, nous irions chercher hors de l'éco-système l'essentiel de ce qui fait la science. Sujet transcendental, apriorisme, introspection, conventionnalisme, mutationnisme sont autant de mots qui désignent la source endogène noninteractive d'une construction dont on pourrait décrire la structure avant qu'elle ne se fasse, donnée avant que d'apparaître. Se référant d'ailleurs aux seules leçons philosophiques qu'il semble avoir reçues, Piaget refuse de croire aussi bien à l'existence d'un fait non relié à une expérience sur le monde qu'à une quelconque faculté qui opérerait en dehors du cadre de l'expérience. La genèse

4. Ibid., pp. 84-85. 
qu'il décrit alors de la nécessité logique - ou des lois de la pensée - à partir des états inférieurs d'équilibration sensori-motrices aboutit fort bien à démystifier cette apparente autonomie de la pensée face à un monde qu'elle rencontrerait et qui aurait, lui aussi, ses lois propres. Il n'y a que les lois du monde construites en même temps qu'apparaissent celles de la pensée, et elles ne peuvent que coïncider. Et si elles sont nécessaires et universelles, c'est que l'éco-système cognitif a, par définition, les dimensions de l'univers et se ferme.

L'embarras cartésien, issu de la science nouvelle et qui le conduit à séparer totalement le sujet de l'objet, trouve ainsi sa solution, non dans une analyse plus poussée des conditions subjectives de la connaissance, mais dans l'abolition - méthodologiquement absolue - de leur indépendance, sans en confondre jamais cependant les notions ni réduire la pensée à la matière.

Si nous résumons les deux cas étudiés, celui de l'origine sensorielle des connaissances et celui de tous les apriorismes, nous voyons que dans le schéma sujet-objet, organisme-milieu, il y a une zone d'interaction hors de laquelle Piaget se refuse à regarder. Les structures cognitives et les structures de i'objet sont saisies, dans leur devenir même, à l'intérieur d'une structure d'interaction. Nous sommes contraints de conclure en disant qu'aux questions traditionnelles "comment sommes-nous intelligents?" et " comment le monde est-il intelligible? " il a substitué celle-ci : "comment en arrivons-nous à avoir un comportement adapté intelligemment au monde?" c'est-à-dire selon ce critère d'équilibre qu'est la réversibilités.

Il se:ait inconvenant ici de contester la validité de cette approche dont les preuves d'efficacité se sont révélées irréfutables, surtout au niveau des analyses psychologiques qui l'ont engendrée. Je formule cependant deux remarques: 1) Piaget a dressé un tableau des épistémologies avec les théories psychologiques et biologiques correspondantes. Mais il me semble que le choix même des caractéristiques ${ }^{6}$ à faire entrer deux à deux dans les six cases doit aboutir nécessairement au classement proposé, et où Piaget et son constructivisme figurent au terme comme un achèvement :

PIAGET, J., La psychulogie de l'intelligence, Colin, Paris, 1967, p. 17.

6. Prépondérance de l'objet, du sujet, interaction; génétique, non génétique. 
chaque fois qu'il place une épistémologie traditionnelle ici plutôt que là, il ne peut la retenir toute, mais simplement d'elle cet aspect qui révèle une plus ou moins grande préoccupation systématique, ou son absence totale. Je suis enclin $\grave{a}$ croire que la distance que Piaget peut prétendre établir ainsi entre lui et les "autres " comporte aussi son illusion. Le développement même du savoir devrait nous conduire à chercher la première structure piagétienne fondamentale chez les présocratiques, et il ne peut que l'élargir pour englober des faits nouveaux. $A$ vrai dire, seul Descartes apparaît comme une antithèse : aussi ne figure-t-il pas au tableau !

2) Par nature, l'approche piagétienne privilégie les perceptions globales en éliminant les questions de frontières :

"Il reste cependant possible de définir l'intelligence par la direction dans laquelle est orientée son développement, sans insister sur les questions de frontières ...." ${ }^{7}$

Toute la fécondité de l'approche systémique est à ce prix, mais elle élimine du coup la possibilité même d'une prise de position ontologique que nous savons avoir toujours sous-tendu toute épistémologie philosophique. Je me demande : si, en dehors de l'être de l'interaction expérimentable, il y a de l'être, peut-être que la fermeture du système n'est qu'illusion, et qu'un perpétuel déséquilibre viendra féconder la raison humaine en l'obligeant à toujours recommencer?

Du moins, il ne m'apparaît pas, jusqu'ici, que le paradoxe de la connaissance, si bien dit par Heidegger, soit levé : " elle est apportée dans sa propre lumière ", ou "elle n'est pas à la maison dans sa propre maison ". Il reste que c'est toujours du sujet dont on dit qu'il connaît le monde et non l'inverse, et, comme tel, il en est toujours séparé, même si j'emboîte bien les étapes de sa rencontre avec le monde.

Faculté de Philosophie, Université Laval.

7. Ibid., p. 16 